\title{
The application of the CEP in control charts to diagnose critical points in baking processes in an Industrial Pole of Manaus Company
}

\author{
Sidney dos Santos Oliveira ${ }^{1}$; Rosimeire Freires Pereira Oliveira $^{2}$. \\ ${ }^{1}$ Manaus-Amazonas-Brasil. (sidneyoliveira54@yahoo.com.br) \\ ${ }^{2}$ Manaus-Amazonas-Brasil.(rosimeirefpol@yahoo.com.br)
}

\begin{abstract}
The diagnosis of the critical points for correction through a plan of action and elimination of defects are strategic issues for the quality of the product. The purpose of this article is to show the use of Statistical Process Control (CEP) as an important tool to diagnose critical points in the production process. The methodology was based on the integration of control charts analysis through the CEP applied in monitoring the cooking process. The proposed approach combines graphics for troubleshooting and views to be used to process performance visualization. The methodology used was observation collection and direct data developed in the form of case study on a Polo Industrial Company Manaus (PIM). The results showed variability shown by statistical charts, making possible better visualization and diagnosis of the critical points to possible corrections and improvements in process productivity, making possible an increase in quality and profitability.
\end{abstract}

Keywords: CEP, control charts, critical points, variability.

\section{A aplicação do CEP nas cartas de controle para diagnosticar pontos críticos nos processos de estufagem em uma Empresa do Polo Industrial de Manaus}

\section{RESUMO}

O diagnostico de pontos críticos para correção através de um plano de ação e eliminação de defeitos são questões estratégicas para a qualidade do produto. O objetivo do artigo é mostrar o uso do Controle Estatístico do Processo (CEP) como ferramenta importante para diagnosticar pontos críticos em processos produtivos. A metodologia foi baseada na integração de análise das cartas de controle por meio do CEP aplicado no monitoramento do processo de estufagem. A abordagem proposta combina gráficos para localização de defeitos e visualizações, sendo usados para visualização do desempenho do processo. A metodologia utilizada foi obervação direta e coleta de dados, desenvolvido sobre forma de estudo de caso em uma empresa do Polo Industrial de Manaus (PIM). Os resultados apontaram uma variabilidade demonstrada através de gráficos estatísticos, possibilitanto uma melhor visualização do processo, e diagnóstico dos pontos críticos para possíveis correções e melhorias da produtividade, possiblitando um aumento na qualidade e lucratividade da empresa.

Palavra- Chaves: CEP, cartas de controle, pontos críticos, variabilidade.

\section{INTRODUÇÃO}

O aumento da competitividade de mercado, tem levado as empresas a buscarem métodos que auxiliam no diagnóstico de pontos críticos para a aplicação de ações corretivas e melhorias contínuas. O CEP é uma ferramenta que a muitos anos tem auxiliado no diagnóstico de falhas. Hoje em dia já se usam mecanismos tecnológicos para verificação de variabilidade, mas, é dentro dos conceitos do CEP é que se pode ter uma visão dos processos se estão dentro ou fora de controle.

O CEP pode até ser considerado ultrapassado por algumas empresas, entretanto, ainda é uma ferramenta muito eficaz para diagnosticar variabilidade nos processos industriais, que além de demonstrar os pontos de inconsistência, traz também à causa do problema [1]. 
Sidney dos Santos Oliveira; Rosimeire Freires Pereira Oliveira / ITEGAM-JETIA Vol.02, No 06, pp.61-67. Junho, 2016.

Detectar problemas em processos são estratégias para a qualidade de um produto, o uso do CEP nas análises das cartas de controle. Buscando uma abordagem que combina com gráficos para localização de defeitos e visualizações, sendo usados para ilustrar o desempenho.

O objetivo da aplicação do CEP nas cartas de controle é evidenciar as variabilidades ocorridas durante um processo, possibilitando o desenvolvimento de gráficos que possibilitam uma visão mais crítica dos pontos de inconsistência [2].

O CEP permite apontar as variabilidades demonstradas através de representações gráficas, possibilitando uma melhor visualização do processo, com intuito de demonstrar possíveis falhas, para aplicação de correções mais eficientes, na busca da melhoria contínua.

O estudo do CEP nas análises das cartas de controle, para diagnosticar pontos críticos dos processos produtivos, em qualquer que seja o seu segmento mercadológico: indústria, agroindústria, comércio ou serviço, tem bastante relevância não só para o atendimento das necessidades dos clientes como também para redução dos custos e melhoria continua nos processos, trazendo vantagem competitiva, além de rentabilidade para a sobrevivência organizacional [3].

Em outras palavras, o presente estudo busca trazer a importância da aplicação do CEP nas análises das cartas de controle não só como método corretivo, mas principalmente, como preventiva pois possibilita diagnosticar pontos críticos. Diante do exposto, definiu-se o problema de pesquisa: De que forma o CEP contribui nas análises das cartas de controle para diagnosticar pontos críticos?

\section{REVISÃO BIBLIOGRÁFICA}

A tecnologia surgiu para atender os novos conceitos de produtividade. $\mathrm{O}$ monitoramento variável mais comum é $T B E$ (tempo entre os eventos). Embora este termo seja utilizado num contexto mais amplo, representa o tempo entre falhas consecutivas de equipamento ou sistema. As variabilidades são consideradas como alterada pela natureza inseparável do processo normal. Já as variações de causa específica são definidas como variações anormais de processo, que devem ser investigados [4].

O CEP é um instrumento criado a partir de cálculos estatísticos, que representam o comportamento de uma determinada técnica, usadas para monitorar a variabilidade do processo e avaliar sua estabilidade. Ela nos permite apontar qual o tipo de variação que esta atuando no processo num apurado período. Isto caracterizará se o processo estará ou não sob controle estatístico [5].

O CEP tem como base inicial a coleta de dados seguindo com outros instrumentos utilizados pela qualidade. Através dessa coleta é possível realizar uma análise criteriosa sobre o que foi encontrado [6].
A importância da ferramenta pauta-se nas diversas aplicações que bem utilizada traz vantagens que tende a melhorar a qualidade do sistema da organização. Bem como, melhorar os procedimentos para que se possa ter uma qualidade nos serviços oferecidos. Através da análise do CEP pode-se ter, processos com menos variabilidade, com níveis de qualidade no resultados final. E surpreendentemente quando se fala em melhorar processos, isso significa que, não somente a melhorar a qualidade, mas também diminui os custos.

A junção dos processos de produtivos de objetos relativos às configurações não-aleatórias foram constituídas para promover a detecção de variações referentes a causas específicas e preparados a partir das características da distribuição normal, através de regras estatísticas relativamente simples.

Verificar pontos fora dos limites de controle é uma atividade simples, entretanto, identificar configurações nãoaleatórias é uma tarefa que gera muitas dúvidas e que requer uma pesquisa minuciosa, daí a utilização do CEP para diagnóstico e monitoramento [7].

Os Gráficos de controle são ferramentas gráficas que auxilia no monitoramento e diagnostico dos desempenhos de um processo ao longo do tempo, detectando possíveis mudanças de grandeza nos valores nominais dos principais parâmetros; tais como desvio médio padrão ou de um desempenho variável descritiva [8].

Para a construção dos gráficos de controle para variáveis deve-se observar os passos a seguir [9]:

$\checkmark$ Sugerir o processo a ser controlada;

$\checkmark$ Coletar os dados a partir das amostras retiradas do procedimento;

$\checkmark$ Coletar as amostras tipo n, cada uma contendo $\mathrm{n}$ dividida em suas propriedades;

$\checkmark$ Coletar as amostras em intervalos contínuos e registrar as observações na ordem em que foram obtidas;

$\checkmark$ Construir a escala e traçar as linhas centrais e os limites de controle nas cartas.

Após a construção dos gráficos de Controle, possibilita observar como o processo está se comportando durante um determinado período de tempo. Essa informação sobre a estabilidade do método permite avaliar se o procedimento está SOB CONTROLE ou FORA DE CONTROLE, assim fazer um diagnostico mais preciso da situação.

Nos gráficos de controle, deve-se marcar o eixo vertical do lado esquerdo com os valores $\mathrm{x}, \mathrm{R}$, s ou AM conforme o par de gráficos escolhidos; Marcar os pontos no gráfico; Interpretar os gráficos construídos; Averiguar andamento do processo observando se o mesmo está sob controle estatístico; Verificar se o estado de controle é adequado ao processo; Revisar periodicamente os valores dos limites de controles [9]. 
Sidney dos Santos Oliveira; Rosimeire Freires Pereira Oliveira / ITEGAM-JETIA Vol.02, No 06, pp.61-67. Junho, 2016.

Esta explicação é realizada de forma oposta, ou seja, é necessário conhecer os padrões de distribuição que evidenciam a 'falta de controle' de um determinado processo, distinguindo-se estes padrões. Visto que, um processo só estará FORA DE CONTROLE se apresentar pontos fora dos limites de controle e/ou uma configuração especial. Essas configurações, que indica a presença de variação especial e, por tanto, a falta de controle do processo.

É importante ter um domínio dos processos, a utilização das cartas de controle é essencial para a aplicação do CEP, caso sua análise não seja feita de forma eficiente poderá acarretar problemas futuros.

Observando a necessidade de seguir uma sequência de passos para as análises das cartas de controle de forma que possibilite um diagnóstico mais preciso. Segundo [7] sugere uma sequência de passos para análise das cartas de controle tais como:

Estabelecer limites de controle experimentais após a coleta de dados de acordo com o Planejado para a Amostragem;

Examinar se todos os pontos estão dentro dos limites de controle e se nenhum limite está em discordância.

Averiguar se nos passos anteriores está tudo conforme os procedimentos estabelecidos, caso as duas condições forem satisfeitas, pode-se dizer que o processo está sob controle estatístico, e o próximo passo é avaliar a capacidade do processo.

$\mathrm{Na}$ eminência de haver pontos fora dos limites de controle e/ou algum procedimento em discordância, pode-se dizer que, o processo está fora da condição de controle estatístico. Neste caso, deve-se fazer um diagnostico para identificar a possível incoerência em cada ponto.

Após a identificação das causas do não conforme, devem-se eliminar tais pontos da amostragem e novos limites experimentais devem ser calculados e analisados novamente. Esses passos devem ser executados até que se conclua que o processo está fora ou sob controle estatístico.

Ao seguir cada passo, pode proporcionar uma diminuição das falhas ao analisar as cartas de controle, se os passos forem executados continuamente, podem restar poucos pontos para análise, o que diminui a representatividade do processo, para essa situação a solução é coletar novas amostras e reiniciar a análise.

Após construir os gráficos de Controle, deve-se observar como o processo está se comportando durante um determinado período de tempo, essas informações sobre a estabilidade do método permite avaliar se o procedimento está SOB CONTROLE ou FORA DE CONTROLE. Mas, é necessário conhecer os padrões de distribuição que evidenciam a 'falta de controle' de um determinado processo, distinguindo-se estes padrões.
Ao conhecer as amostras de variação que evidenciam a falta de controle, as avaliações devem ser feitas no gráfico original tentando achar pontos fora dos limites de controle e/ou alguma configuração especial. Caso seja encontrado, o processo analisado será dito 'fora de controle' e, não havendo nenhuma nem outra configuração, o processo será dito 'sob controle estatístico'.

\section{MATERIAIS E MÉTODOS}

A pesquisa foi feita por meio de uma pesquisadiagnóstico através de um estudo de caso em uma empresa do Polo Industrial de Manaus (PIM) com o intuito de analisar a importância da utilização do CEP nas cartas de controle em processos produtivos.

A pesquisa tem natureza qualitativa e quantitativa, buscando estabelecer relações entre as cartas estudadas que são os quantitativos de controle com os pontos de variabilidades das operações produtivas e também investigar e comparar os pontos críticos correlacionando-os aos processos inerentes ao funcionamento de uma organização.

No que tange à finalidade da pesquisa, pode-se dizer que, o estudo se caracteriza como descritiva, por buscar descrever a correlação entre as cartas de controle das operações produtivas com o CEP aplicado para demonstração de variabilidade e o impacto advindo desta relação.

A pesquisa foi literária, documental e de campo por meio de coleta de dados e observação direta oportunizada em visitas técnica de campo. As informações fornecidas para pesquisa e que foram utilizadas como fonte de informação relevante ao estudo de caso valorizando as evidências oriundas de outras fontes foi: Cartas e Relatórios de Controle além de dados com planilhas mensais e gráficos. Todos esses documentos fornecidos foram analisados e trabalhados para montar a pesquisa.

Para a pesquisa bibliográfica foram utilizados materiais publicados por autores renomados, que já obtiveram resultados em pesquisas semelhantes, como artigos científicos, livros editorados e até teses de doutorado; Na pesquisa documental foram analisados os resultados das cartas de controle.

Os dados foram coletados através de: questionários, entrevistas, formulários, relatórios, cartas de controle, dados retirados do SI, e que foram pesquisados no período 02/01/2016 à 30/04/2016. As análises dos dados coletados no processo foram realizadas através de tabelas e interpretação dos gráficos de controle que representam a forma da metodologia avaliada, ou seja, o tipo de variação que ocorreu no processo no período estudado.

Obtidas através dos calculos dos:

- Gráficos da média $-\bar{x}=\frac{1}{n} \sum_{i=1}^{n} x_{i}=\frac{x_{1}+x_{2}+\ldots+x_{n}}{n}$ 
Sidney dos Santos Oliveira; Rosimeire Freires Pereira Oliveira / ITEGAM-JETIA Vol.02, Nº 06, pp.61-67. Junho, 2016.

- Gráfico de amplitude- R: $x_{\text {maior }}-x_{\text {menor }}$

- Gráfico desvi-padrão-s: $s_{i}=\sqrt{\frac{1}{n-1} \sum_{j=1}^{n}\left(x_{i j}-\bar{x}_{i}\right)^{2}}$

E para calcular os limites de controle LSC e LIC foram utilizados os gráficos da média. Através de valores representados por pontos no gráfico, através de duas linhas horizontais: os limites de controlo superior $(L S)$, representa o deslocamento $D>$ 0 , e inferior ( $L I)$, para $D<0$.

Sendo inferidas as configurações que evidenciam a 'falta de controle', buscou-se encontrá-las no gráfico que representa o processo simulado. Baseado nestas informações, concluindo assim, sobre a estabilidade ou não do processo.

\section{RESULTADOS E DISCUSSÕES}

Após as peças passarem pelo processo de estufagem são feito a inspeção do dimensionamento e seguido da montagem das cartas de controle, para que possa verificar se o processo está sob controle ou não. Na Tabela 1, são apresentados os dados obtidos através das cartas de controle com especificação em $(\mathrm{mm})$ de Limite Inferior (LI) 1,40 mm e Limite Superior (LS) 1,60 mm.

Em seguida foram montadas classes e frequência da carta de controle demonstradas na Tabela 2 contendo 7 classes.

Tabela 1 - Tabela de Frequência.

\begin{tabular}{r|r|r|r}
\multicolumn{1}{c|}{ Classe } & & \multicolumn{1}{c|}{ Bloco } & \multicolumn{1}{|c}{ Freqüência } \\
\hline 1 & 0,86 & 69,97 & 0 \\
\hline 2 & 1,09 & 76,05 & 2 \\
\hline 3 & 1,31 & 82,14 & 11 \\
\hline 4 & 1,53 & 88,22 & 20 \\
\hline 5 & 1,75 & 94,30 & 18 \\
\hline 6 & 1,97 & 100,38 & 9 \\
\hline 7 & 2,19 & 106,46 & 0 \\
\hline & & Mais & 0 \\
\hline
\end{tabular}

Fonte: Empresa do PIM (2016).

Na Tabela 1, foi montado o gráfico de frequência após o levantamento da carta. Para simplificação dos dados e uma melhor visualização.

Tabela 2- Dados das entregas e devoluções das placas KSSJ de Jan a abr de 2016.

\begin{tabular}{l|r|r|r|r|r}
\hline \multicolumn{1}{c|}{ KSSJ } & \multicolumn{1}{c|}{ JAN } & \multicolumn{1}{c|}{ FEV } & \multicolumn{1}{c|}{ MAR } & \multicolumn{1}{c|}{ ABR } & TOTAL \\
\hline ENTREGA & 47.760 & 56.080 & 52.644 & 54.228 & 210.732 \\
\hline DEVOLUÇÃO & 7.654 & 8.256 & 13.657 & 10.450 & 40.017 \\
\hline INDICE NG & $16,02 \%$ & $14,72 \%$ & $25,94 \%$ & $19,278 \%$ & $18,99 \%$ \\
\hline
\end{tabular}

Fonte. Empresa do PIM (2016).
$\mathrm{Na}$ Tabela 2, observa-se as quantidades de entregas e devoluções das peças KSSJ, haja vista que, caso o processo venha estar sob controle esse índice é cada vez menor, e as falhas são menos usuais. Entretanto, só é possível obter um processo sob controle quando se tem uma análise eficiente e ferramentas eficazes, por isso que, o CEP, torna-se um mecanismo de uma grande eficiência para que a produção se mantenha sob controle.

As empresas precisam evitar os desperdício, e redução dos custos, quando se utiliza o CEP, nas análises das cartas de controle, a probabilidade de ocorrerem erros são menores, pelo fato de que os diversos métodos do CEP, só tende a buscar as mudanças nas variabilidades, em um varredura dos processo produtivos.

Tabela 3 - Peças produzidas e perda da placa KSSJ.

PEÇAS PRODUZIDAS E PERDA DA PLACA KSSJ

\begin{tabular}{|c|c|c|c|c|}
\hline $\begin{array}{c}\text { TURN } \\
\text { O }\end{array}$ & $\begin{array}{l}\text { TOTAL } \\
\text { PROD. }\end{array}$ & $\begin{array}{c}\text { TOTAL } \\
\text { REFUGAD } \\
\mathrm{O}\end{array}$ & $\begin{array}{c}\text { INDIC } \\
\text { E } \\
\text { PERDA }\end{array}$ & DATA \\
\hline $1^{\circ}$ & 760 & 340 & $44,73 \%$ & \multirow{3}{*}{$01 / 04 / 2016$} \\
\hline $2^{\circ}$ & 1566 & 354 & $22,60 \%$ & \\
\hline $3^{\circ}$ & 1510 & 410 & $27,15 \%$ & \\
\hline $1^{\circ}$ & 1740 & 180 & $10,34 \%$ & \multirow{3}{*}{ 02/04/2016 } \\
\hline $2^{\circ}$ & 1426 & 144 & $10,09 \%$ & \\
\hline $3^{\circ}$ & 1266 & 554 & $43,75 \%$ & \\
\hline $1^{\circ}$ & 1830 & 90 & $4,91 \%$ & \multirow{3}{*}{ 03/04/2016 } \\
\hline $2^{\circ}$ & 322 & 58 & $18,01 \%$ & \\
\hline $3^{\circ}$ & & & & \\
\hline \multicolumn{5}{|c|}{ TOTAL ACUMULADO } \\
\hline $1^{\circ}$ & 4.330 & 610 & $14,08 \%$ & \multirow{4}{*}{$01 / 04 / 2016$} \\
\hline $2^{\circ}$ & 3.314 & 556 & $16,77 \%$ & \\
\hline \multirow[t]{2}{*}{$3^{\circ}$} & 2.776 & 964 & $34,72 \%$ & \\
\hline & $\begin{array}{l}10.420 \\
\text { Peças } \\
\text { Prod. }\end{array}$ & $\begin{array}{c}2.130 \\
\text { Refugadas }\end{array}$ & $20,44 \%$ & \\
\hline
\end{tabular}

Fonte: Empresa do PIM (2016).

Conforme observado na Tabela 3, a empresa esta tendo um índice de refugo em torno de $20,44 \%$, de 10.420 peças produzidas 2.130 peças estão tendo refugos, índices considerados bem significantes para empresa. Sugere-se que, a verificação do CEP venha ser ampliada para os requisitos de inspeção utilizados pelo cliente final.

\section{VI.1 O USO DO CEP NAS ANÁLISES DAS CARTAS DE CONTROLE}

Como já observado o CEP traz uma análise mais visual e com maior alcance nas causas, por demonstrar com uma maior 
Sidney dos Santos Oliveira; Rosimeire Freires Pereira Oliveira / ITEGAM-JETIA Vol.02, No 06, pp.61-67. Junho, 2016.

eficiência o andamento do processo. O CEP ao ser aplicado nas cartas de controle traz um parâmetro do andamento da produção através das demonstrações gráficas, de forma que, ao analisar, pode-se observar se os resultados as inspeções estão dentro ou fora dos limites de controle. Apresentando um resultado final do processo produtivo, se está sob ou fora de controle, para que possa aplicar uma ação corretiva mais precisa, e para que isso ocorra é necessário conhecer o andamento da produção.

As figuras 1,2 e 3, mostram a aplicação do CEP nas cartas de controle demostrada nas Tabelas 1, 2, 3 e 4.
Conforme visto na Tabela 4, a média e o desvio padrão auxilia na análise das cartas de controle utilizando o CEP. Proporcionando um parâmetro Aceitável e Inaceitável, para que o diagnóstico do processo possa ser mais preciso.

Ao analisar as Figuras 1, 2 e 3, percebeu-se que, os dados alimentados nas cartas de controle, após o processo de estufagem, encontram-se fora dos limites de controle, demonstrando umas grandes variabilidades, de forma que o desvio padrão ficou em torno de 0,22 e a média em 1,53. Visto que, a dimensão é de $1,5 \pm 0,1 \mathrm{~mm}$. Foram copiladas 7 classes, com desvio padrão de 0,03 entre as classes e com intervalo de 6,08 entre os blocos, conforme apresentado no histograma da figura 30 .

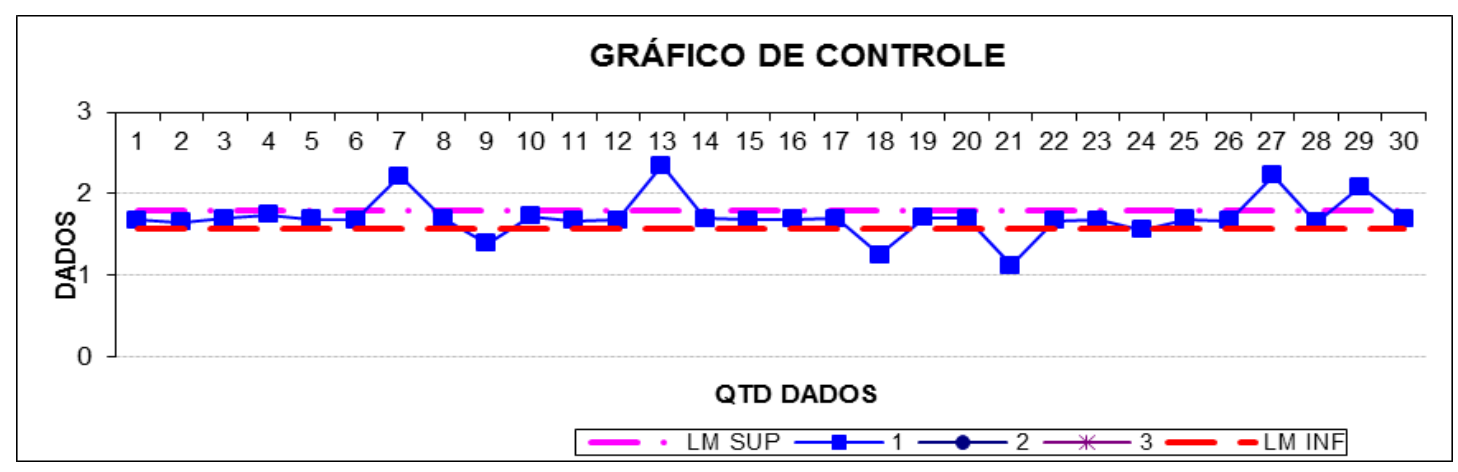

Figura 1- Gráfico de Controle após as peças passarem pelo processo de estufagem.

Fonte: Empresa do PIM (2016).

Na Figura 1, observa-se o gráfico de controle com o LI e LS, onde os registros gráficos se encontram fora de controle, decorrente dos pontos que não estão dentro dos parâmetros de aceitação.
Mostrando-se que, o gráfico de controle dá uma visão do processo, que permite averiguar se o processo está sob controle ou não.

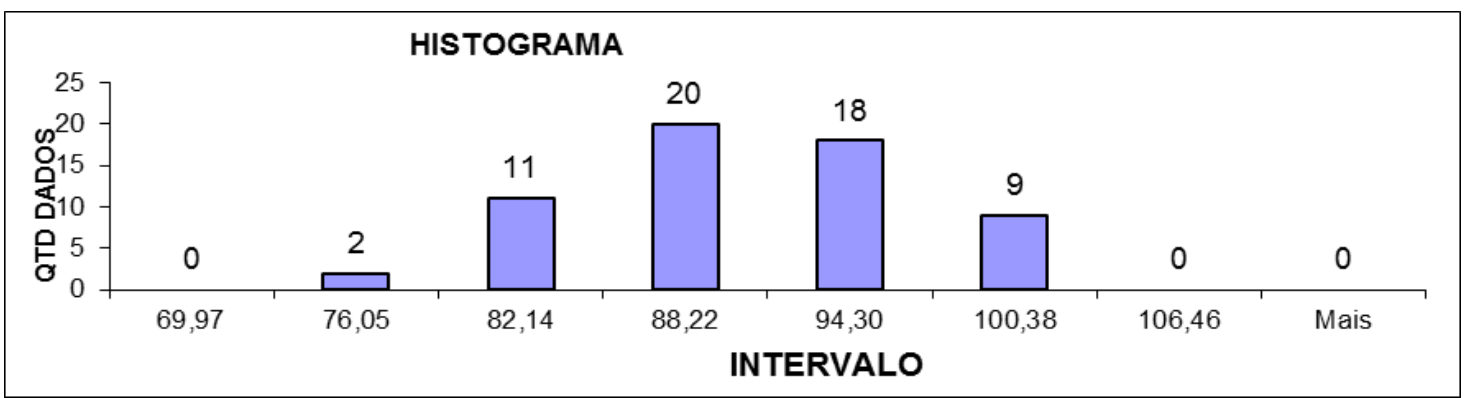

Figura 2- Histograma das classes após as peças passarem pelo processo de estufagem.

Fonte: Empresa do PIM (2016).

Colocando os dados das cartas de controle no histograma, verifica-se uma visualização do processo em outro formato, a mesma visualização dos resultados sob outro formato, utilizando as classes e suas frequências.
Mostrando dessa forma que ambos gráficos são muito eficientes para o diagnostico dos pontos fora de controle considerado críticos. Após a aplicação dos gráficos de controle observou-se que o processo se encontra inaceitável para os parâmetros de qualidade. 
Sidney dos Santos Oliveira; Rosimeire Freires Pereira Oliveira / ITEGAM-JETIA Vol.02, No 06, pp.61-67. Junho, 2016.

\begin{tabular}{|c|c|c|c|c|}
\hline MEDIA & 1,53 & \multirow{2}{*}{ CPX } & 0,11 & INACEITÁVEL \\
\cline { 1 - 2 } DESVPAD & 0,22 & & & \\
\hline
\end{tabular}

Figura 4- Média e desvio padrão após a análise da carta de controle das dimensões após estufagem.

Fonte: Autores, (2016).

Ao aplicar CEP nas analises das cartas de controle, pode-se observar que, o maior problema concentrou-se no processo de estufagem que tem causado um refugo com perdas consideradas prejuízos para a empresa.

O CEP, dá um parâmetro mais preciso do ponto de variabilidade e tolerância. Proporciona um resultado dos processos com mais eficácia, na busca de eliminar falhas e diagnosticar pontos críticos.

Sugere-se que, a empresa faça uma verificação no processo de estufagem, visto que, o processo que monstra a não conformidade está após a peça passar pela estufa.

\section{CONCLUSÃO}

O CEP se mostrou um instrumento muito eficiente no diagnostico de pontos críticos, atendendo o objetivo proposto, por permitir auxiliar na visualização da causa raíz, permitindo uma ação corretiva mais eficaz.

Após a aplicação do CEP na empresa em estudo, pode perceber que, a maior variação dos pontos fora de controle estava centralizada nas devoluções das peças de resina termoplásticas. Demonstrando que o CEP, tras uma visualização mais clara dos problemas no proceso monitorado em curto intervalo de tempo utilizando um menor número de amostragem.

Outro ponto abordado foi o valor médio deste processo, após ter sido detectada uma situação fora de controle com a demonstração de tabelas e gráficos possibilitou ver uma vantagem económica decorrente da rapidez do diagnostico.

Olhando sob o ponto de vista estatístico, a análise das cartas de controle através do CEP, demonstrou-se importante por ter apresentado através das tabelas e gráficos a sinalização de ocorrência de uma causa especial, que foi o índice de devolução em determinados períodos.

Já sob o ponto de vista econômico, uma análise eficiente pode ocasionar uma redução nos custos relacionados as diminuição das devoluções de produtos entregues ao cliente final, assim como, redução nas perdas ocasionadas pela não conformidade dos produtos manufaturados.

A utilização do CEP nas cartas de controle se torna importante sendo empregado como métodos estratégicos, para a melhoria contínua, servindo para aperfeiçoar os resultados, mantendo-se nos níveis desejados, caso contrário, tornar-se-ão apenas mais o fator de custo que não agrega valor algum ao produto ou processo.

E que a diminuição dos prejuízos, obtidos por meio de sinalização mais precisa, considera-se imprescindível, pois, todos os planejamentos internos ou externos à organização, permaneçam devidamente integrados e alinhados com as políticas e os objetivos da empresa. Dessa forma, o sucesso ou fracasso das análises das cartas de controle, dependerá de um bom método.

A utilização do CEP para diagnostico de pontos críticos através da investigação de limites de controle dependerá de uma avaliação lógica de todo o sistema, cabendo ao analista a responsabilidade indelegável para o alcance dos resultados.

\section{AGRADECIMENTOS}

Ao Instituto de Tecnologia e Educação Galileu da Amazônia (ITEGAM), ao PPGEP do Instituto de Tecnologia Universidade Federal do Pará (ITEC-UFPA).

\section{REFERÊNCIAS}

[1] MONTGOMERY, D.C., Statistical quality control: a modern introduction. International Student Version 7th edition. Wiley, 2012.

[2] OLIVEIRA, Sidney dos Santos.; LEITE, Jandecy C.; SOUZA, José A. da S., OLIVEIRA, Rosimeire Freires Pereira. Uso do Controle Estatístico de Processo (CEP) na gestão de operações produtivas em uma indústria no Polo Industrial de Manaus. Revista SODEBRAS. Volume $10 \quad \mathrm{~N}^{\circ} 117$ de julho/2015.

[3] RIBEIRO, J. L. \& CATEN, C. T. Controle Estatístico do Processo. Apostila do Curso de Pós-Graduação em Engenharia de Produção. Porto Alegre: Editora da UFRGS, 1998.

[4] CHENG, Zhi-Qiang, Yi-Zhong, Bu Jing, Song Hua-Ming. Mean Shifts Diagnosis And Identification In Bivariate Process Using Ls-Svm Based Pattern Recognition Model. International Journal Of Industrial Engineering. 20(7-8), 453467. 2013.

[5] ROCCO, J. Perla; LLOYD, P. Provost; SANDY, K. Murray; The run chart: a simple analytical tool for learning from variation in healthcare processes; BMJ Qual Saf, 20: 46-51. 2011.

[6] MACHADO, José Fernando. Método Estatístico: Gestão de Qualidade para Melhoria Continua. São Paulo: Saraiva, 2010.

[7] WERKEMA, Maria Cristina Catarino. Avaliação de sistemas de medição. 2.ed. Belo Horizonte: Werkema. 2012. 
[8] REYNOLDS Jr., M.R., LOU, J.Y., An evaluation of a GLR control chart for monitoring the process mean. Journal of Quality Technology 42. 287-310. 2010.

[9] LIM, Sarina Abdul Halim; ANTONY, Jiju; GARZA-REYES, Jose Arturo; ARSHED, Norin. Towards a conceptual roadmap for Statistical Process Control implementation in the food industry. Trends in Food Science \& Technology xx. 1e13. 2015. 